\title{
Comparison of the effect of general and spinal anesthesia for elective cesarean section on maternal and fetal outcomes: a retrospective cohort study
}

\section{Tae-Yun Sung ${ }^{1,2}$, Young Seok Jee ${ }^{1}$, Hwang-Ju You' ${ }^{1}$, and Choon-Kyu Cho'}

${ }^{1}$ Department of Anesthesiology and Pain Medicine, ${ }^{2}$ Myunggok Medical Research Center, Konyang University Hospital, Konyang University College of Medicine, Daejeon, Korea
Received September 2, 2020

Revised October 12, 2020

Accepted October 15, 2020

\section{Corresponding author}

Young Seok Jee, M.D., Ph.D.

Department of Anesthesiology and

Pain Medicine, Konyang University

Hospital, Konyang University College

of Medicine, 158 Gwanjeodong-ro,

Seo-gu, Daejeon 35365, Korea

Tel: 82-42-600-9319

Fax: 82-42-545-2132

E-mail: jisaac@naver.com
Background: Anesthesia is needed to ensure both maternal and fetal safety during cesarean sections. This retrospective cohort study compared maternal and fetal outcomes between general and spinal anesthesia for cesarean section based on perioperative hemodynamic parameters (pre- and postoperative systolic blood pressure, heart rate), mean difference of hematocrit and estimated blood loss, and neonatal Apgar scores at 1 and 5 min.

Methods: Data from electronic medical records of 331 singleton pregnancies between January 2016 and December 2018 were analyzed retrospectively; 44 cases were excluded, and 287 cases were assigned to the general group $(n=141)$ or spinal group $(n=146)$.

Results: Postoperative hemodynamic parameters were significantly higher in the general group than the spinal group (systolic blood pressure: $136.8 \pm 16.7$ vs. $119.3 \pm 12.7 \mathrm{mmHg}$, heart rate: $93.2 \pm 16.8$ vs. $71.0 \pm 12.7$ beats $/ \mathrm{min}$, respectively, $\mathrm{P}<0.001)$. The mean difference between the pre- and postoperative hematocrit was also significantly greater in the general than spinal group ( $4.8 \pm 3.4 \%$ vs. $2.3 \pm 3.9 \%$, respectively, $\mathrm{P}<0.001)$. The estimated blood loss was significantly lower in the spinal than general group (819.9 \pm 81.9 vs. $856.7 \pm 117.9 \mathrm{ml}, \mathrm{P}<0.001)$. There was a significantly larger proportion of newborns with 5-min Apgar scores < 7 in the general than spinal group (6/141 [4.3\%] vs. 0/146 [0\%], respectively, $\mathrm{P}=0.012$ ).

Conclusions: General group is associated with more maternal blood loss and a larger proportion of newborns with 5-min Apgar scores $<7$ than spinal group during cesarean sections.

Keywords: Cesarean section; General anesthesia; Outcome measures; Spinal anesthesia.

\section{INTRODUCTION}

Anesthesia used for cesarean section is either general or regional. The advantages of general anesthesia include the facilitation of a rapid procedure in obstetric emergencies and loss of consciousness, which ensures less distress to parturient women. The disadvantages of general anesthesia include the possibility of aspiration pneumonia, mater-

This is an Open Access article distributed under the terms of the Creative Commons Attribution Non-Commercial License (http://creativecommons.org/licenses/by-nc/4.0) which permits unrestricted non-commercial use, distribution, and reproduction in any medium, provided the original work is properly cited.

Copyright (C) the Korean Society of Anesthesiologists, 2021 
nal awareness during the operation due to inadequate anesthesia, failed intubation, and respiratory complications in the mother and newborn. Many intravenous anesthetic agents injected into the mother can cross the placental barrier and enter fetal circulation and may cause sedation or respiratory depression of the newborn.

The two types of regional anesthesia used for cesarean sections are spinal and epidural anesthesia. The advantages of regional anesthesia include reduced complications associated with general anesthesia and promotion of initial bonding between the mother and the baby (because the mother is awake during the operation) [1]. Recently, spinal anesthesia has been preferred over epidural anesthesia for cesarean section because of its rapid onset, effectiveness, and lower requirement for local anesthetics; however, it is associated with a higher incidence of arterial hypotension [2]. Spinal anesthesia using small amounts of local anesthetics is less likely to cause maternal systemic toxicity or total spinal anesthesia. Therefore, it is pertinent to compare the effects of general and spinal anesthesia during cesarean sections on maternal and fetal outcomes.

Previous studies have compared postoperative maternal hematocrit (hct) levels between general and spinal anesthesia for cesarean section [3,4]. The Cochrane database [5] has three papers on maternal blood loss in relation to cesarean section; one study has compared epidural and general anesthesia and two studies have compared spinal and general anesthesia.

The Apgar score is an indicator of neonatal well-being. Several studies have reported no significant difference in Apgar scores between general and regional anesthesia [5], but two studies $[6,7]$ reported that the 1-min Apgar scores were lower in general than regional anesthesia. Thus, controversy remains regarding the association of neonatal well-being scores with general and regional anesthesia.

This retrospective study reviewed the medical records of women who underwent cesarean section under general anesthesia or spinal anesthesia and compared the maternal and fetal outcomes based on perioperative hemodynamic parameters (pre- and postoperative systolic blood pressure, heart rate), hematocrit, and estimated blood loss and neonatal Apgar scores at 1 and 5 min between both anesthesia groups.

\section{MATERIALS AND METHODS}

This study was approved by our ethics committee/Insti- tutional Review Board (no. KYUH 2019-04-008) and registered at the Korea Clinical Research Information Service (http://cris.nih.go.kr; no. KCT 0004783). The need for informed consent was waived because of the retrospective study design. We retrospectively analyzed data from 331 singleton deliveries that occurred between January 2016 and December 2018; data regarding maternal and fetal outcomes after general or spinal anesthesia for elective cesarean section were analyzed and compared. Two anesthesiologists were in charge of anesthesia during the obstetric surgeries. Both anesthesiologists induced anesthesia using the same anesthetic agents; patient monitoring, extubation criteria, and the same spinal technique were performed according to our institutional protocol.

All subjects were scheduled for elective cesarean section, with their physical condition classified according to the American Society of Anesthesiologists class 2, since American Society of Anesthesiologists categorizes "pregnancy" as class 2. Exclusion criteria included the need for emergency or epidural anesthesia, conversion from spinal to general anesthesia, and deliveries wherein bleeding was anticipated, such as placenta previa or coagulopathy. In total, 44 of the 331 subjects were excluded. The remaining 287 subjects were classified into either general anesthesia $(\mathrm{n}=141)$ or spinal anesthesia $(\mathrm{n}=146)$ groups (Fig. 1). The anesthesia induction method was dependent on the mother's choice, and the difference in preference between the two anesthesiologists were recorded.

All parturient women fasted for at least $8 \mathrm{~h}$ preoperatively and were not administered any pharmacological premedication. In the operating room, we routinely used standard monitoring, including electrocardiography, noninvasive blood pressure, and pulse oximetry $\left(\mathrm{SpO}_{2}\right)$.

In the general anesthesia group, for all patients, we used

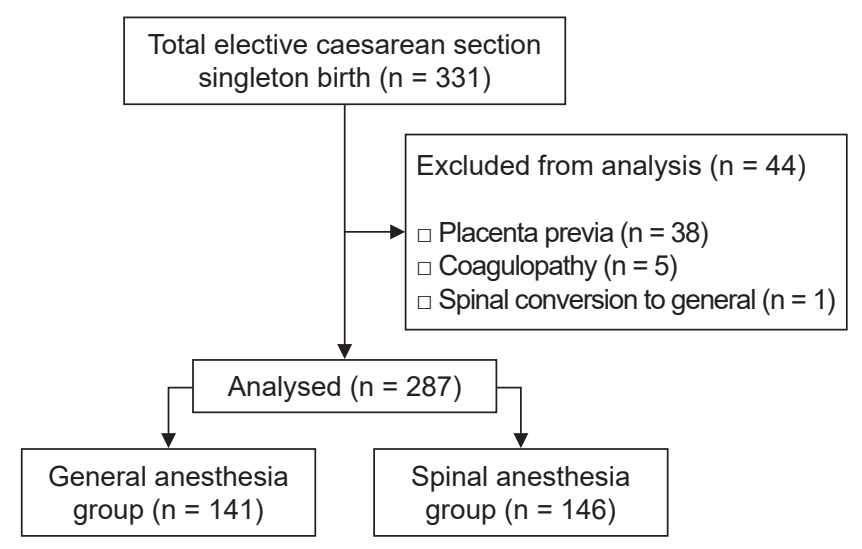

Fig. 1. Flow diagram. 
the bispectral index (BIS) and preoxygenation using $100 \%$ oxygen delivered over 3-5 min. Subsequently, anesthesia was induced with $5 \mathrm{mg} / \mathrm{kg}$ of thiopental. Intravenous injection of $0.5 \mathrm{mg} / \mathrm{kg}$ rocuronium facilitated endotracheal intubation, with the Sellick maneuver applied to prevent aspiration. In all cases, we established controlled ventilation with a tidal volume of $8 \mathrm{ml} / \mathrm{kg}$ and a respiration rate of 12 14 breaths per minute. Anesthesia was maintained with a mixture of $1.5-2.0$ vol\% sevoflurane and $50 \%$ nitrous oxide in oxygen. If a maintenance dose was required, $0.15 \mathrm{mg} / \mathrm{kg}$ of rocuronium was added intravenously. At the end of the surgery, the residual neuromuscular block was reversed with pyridostigmine $(0.2 \mathrm{mg} / \mathrm{kg})$ and glycopyrrolate $(4 \mathrm{\mu g} /$ $\mathrm{kg}$ ). Patients were extubated once they were fully awake to prevent aspiration.

Spinal anesthesia was introduced at L2/3 or L3/4 level with a $25-G$ pencil-point needle (Sprotte, Pajunk, Germany), with patients in a left lateral decubitus position; $\mathrm{O}_{2}$ was supplied at a rate of $6 \mathrm{~L} / \mathrm{min}$ via an oxygen mask. After confirming a clear, free flow of cerebrospinal fluid, 9-10 mg of $0.5 \%$ bupivacaine (Marcaine Heavy, Astra Zeneca, UK) with $10 \mu \mathrm{g}$ fentanyl was injected slowly. Patients were then placed in a fully supine position and tilted $15^{\circ}$ downward and leftward to prevent the supine hypotensive syndrome. The sensory block level was determined using a cold test. The operation was initiated when the sensory block had reached an adequate level (T4-T5). Phenylephrine (1 mg/ h) was continuously infused to prevent arterial hypotension. If hypotension persisted, $0.1 \mathrm{mg}$ phenylephrine was injected by IV bolus, or the continuous injection rate was increased up to $2 \mathrm{mg} / \mathrm{h}$. If hypertension occurred, the continuous injection rate was decreased to $0.5 \mathrm{mg} / \mathrm{h}$ or discontinued. We defined hypotension as systolic blood pressure below $90 \mathrm{mmHg}$ or below $70 \%$ of the baseline blood pressure. After the newborn had been delivered, mothers were sedated with intravenous midazolam as required if she wanted.

Cesarean section was performed via a standard lower segment transverse uterine incision with basic monitoring, including heart rate and blood pressure. After the baby had been delivered, carbetocin (Inj. Hanlim, Korea) was routinely administered to induce uterine contraction.

\section{Measurements}

Data on gestational age, parity, height, body weight, pre and postoperative systolic blood pressure ( $\mathrm{mmHg}$ ), heart rates (beats/min), hct (\%), duration of hospital stay (day), surgical time, anesthesia time, time between skin incision and delivery, estimated blood loss (EBL), and transfusions were collected from medical records. EBL was measured by a visual estimation and a gravimetric method that involves weighing of soiled sponges and measurement of fluid in suction canisters. Newborns were evaluated by a pediatrician in terms of their sex, weight, and 1- and 5-min Apgar scores. Pediatricians were randomly assigned for the cesarean delivery.

We defined our primary outcome as the mean difference between the pre- and postoperative hematocrit, and the secondary outcome as the 1- and 5-min Apgar scores.

\section{Statistical analysis}

The sample size required to detect a statistically significant difference between the pre- and postoperative hematocrit was determined using R software (version 3.5.3, $\mathrm{R}$ Development Core Team, Austria). The differences between the pre- and postoperative hematocrit were measured in a preliminary study ( $\mathrm{n}=10$ for each), and the average difference of hematocrit in the general anesthesia and spinal groups was 5.06 (SD, 3.31) and 2.13 (SD, 4.02), respectively. Sample size was calculated with an effect size of 0.796 , power of $80 \%$, and an $\alpha$-value of 0.05 ; accordingly, 26 subjects were needed in each group.

All statistical analyses were performed using R software (version 3.5.3). The normality of the distribution of continuous variables was analyzed using the Shapiro-Wilk test. We used independent $t$-tests to analyze continuous, normally distributed variables and the Wilcoxon rank-sum test to analyze continuous, non-normally distributed variables. Categorical variables were compared using the $\chi^{2}$ test or Fisher's exact test. Descriptive statistics are presented as mean \pm standard deviation, median $(1 \mathrm{Q}, 3 \mathrm{Q})$, or percentage. Perioperative change in hct (\%) was compared between the two anesthesia groups using repeated-measures analysis of variance, followed by Student's $t$-test. A P value of less than 0.05 was considered statistically significant.

\section{RESULTS}

Between January 2016 and December 2018, a total of 331 women (singleton pregnancies) underwent elective cesarean section at our hospital. In total, 44 patients were excluded owing to failed spinal anesthesia, placenta previa, 
or coagulopathy. Thus, eventually, 287 patients were stratified into either a general anesthesia $(n=141)$ or spinal anesthesia ( $\mathrm{n}=146$ ) groups (Fig. 1).

Demographic data showed no significant differences between the general and spinal anesthesia groups for demographic characteristics, except surgical time (56.9 \pm 13.1 vs. $53.2 \pm 11.1 \mathrm{~min}, \mathrm{P}=0.011$ ) (Table 1).

Maternal and fetal data were as follows: there was no significant difference in preoperative systolic blood pressure between the general and spinal anesthesia groups (136.1 \pm 17.2 vs. $132.1 \pm 17.4 \mathrm{mmHg}$, respectively). However, postoperative systolic blood pressure was significantly higher in the general anesthesia group than in the spinal anesthesia group (136.8 \pm 16.7 vs. $119.3 \pm 12.7$, respectively, $\mathrm{P}<$ 0.001) (Table 2).

Preoperative heart rate was different between the general and spinal anesthesia groups $(81.6 \pm 12.6$ vs. $85.6 \pm 13.9$ beats / $\mathrm{min}$, respectively, $\mathrm{P}=0.011$ ). The postoperative heart rate was significantly higher in the general anesthesia group than in the spinal anesthesia group (93.2 \pm 16.8 vs. $71.0 \pm 12.7$, respectively, $\mathrm{P}<0.001$ ) (Table 2).

The mean postoperative hct level was significantly lower in the general anesthesia group than in the spinal anesthesia group ( $31.4 \pm 3.9 \%$ vs. $34.2 \pm 4.7 \%$, respectively, $\mathrm{P}<$ 0.001). The mean difference between the pre- and postoperative hct level was also significantly greater in the general anesthesia group than in the spinal anesthesia group (4.8 $\pm 3.4 \%$ vs. $2.3 \pm 3.9 \%$, respectively, $\mathrm{P}<0.001$ ) (Table 2 ).

The mean EBL was significantly lower in the spinal anesthesia group than in the general anesthesia group (819.9 \pm 81.9 vs. $856.7 \pm 117.9 \mathrm{ml}$, respectively, $\mathrm{P}<0.001)$. There was no significant group difference in the transfusion rate (ratio of transfused to total subjects) (Table 2).

The proportion of newborns with 1-min Apgar scores < 7 was not significantly different between the two groups, although the general anesthesia group had a significantly larger proportion of newborns with 5-min Apgar scores $<7$ than the spinal anesthesia group (6/141 [4.3\%] vs. 0/146

Table 1. Demographic Data

\begin{tabular}{lccc}
\hline \multicolumn{1}{c}{ Variable } & General $(\mathrm{n}=141)$ & Spinal $(\mathrm{n}=146)$ & P value \\
\hline Age $(\mathrm{yr})$ & $34.1 \pm 4.6$ & $33.5 \pm 4.0$ & 0.214 \\
Height $(\mathrm{cm})$ & $160.3 \pm 5.2$ & $161.1 \pm 5.8$ & 0.258 \\
Weight $(\mathrm{kg})$ & $72.1 \pm 14.9$ & $70.8 \pm 11.2$ & 0.458 \\
Gestation $(\mathrm{wk})$ & $37.0 \pm 2.1$ & $37.3 \pm 1.9$ & 0.298 \\
Gravidity & $3(2,4)$ & $2(1,3)$ & 0.301 \\
Operation (min) & $56.9 \pm 13.1$ & $53.2 \pm 11.1$ & $0.011^{*}$ \\
Anesthesia (min) & $75.0 \pm 14.4$ & $77.5 \pm 12.1$ & 0.114 \\
Skin incision to delivery & $5.9 \pm 2.2$ & $6.2 \pm 2.3$ & 0.305 \\
\hline
\end{tabular}

Values are presented as mean \pm SD or median $(1 \mathrm{Q}, 3 \mathrm{Q})$. ${ }^{\star P}$ value $<0.05$.

Table 2. Maternal and Fetal Parameters

\begin{tabular}{|c|c|c|c|c|c|}
\hline & Measures & General $(n=141)$ & Spinal $(n=146)$ & Mean difference (95\% confidence interval) & $P$ value \\
\hline \multirow[t]{10}{*}{ Maternal } & Preoperative SBP & $136.1 \pm 17.2$ & $132.1 \pm 17.40$ & $4.0(-0.2,8.0)$ & 0.051 \\
\hline & Postoperative SBP & $136.8 \pm 16.7$ & $119.3 \pm 12.7$ & $17.5(14.1,21.0)$ & $<0.001^{\dagger}$ \\
\hline & Preoperative HR (beats/min) & $81.6 \pm 12.6$ & $85.6 \pm 13.9$ & $-4.0(-7.1,-0.96)$ & $0.011 *$ \\
\hline & Postoperative HR (beats/min) & $93.2 \pm 16.8$ & $71.0 \pm 12.7$ & $22.2(18.7,25.7)$ & $<0.001^{\dagger}$ \\
\hline & Preoperative hct (\%) & $36.2 \pm 3.4$ & $36.5 \pm 3.1$ & $-0.3(-1.1,0.4)$ & 0.404 \\
\hline & Postoperative hct (\%) & $31.4 \pm 3.9$ & $34.2 \pm 4.7$ & $-2.8(-3.8,-1.8)$ & $<0.001^{\dagger}$ \\
\hline & dhct & $4.8 \pm 3.4$ & $2.3 \pm 3.9$ & $2.4(1.6,3.3)$ & $<0.001^{\dagger}$ \\
\hline & $\mathrm{EBL}(\mathrm{ml})$ & $856.7 \pm 117.9$ & $819.9 \pm 81.9$ & $36.9(13.2 .60 .6)$ & $0.002 *$ \\
\hline & Transfusion rate (\%) & $3(2.1)$ & $2(1.4)$ & $7.6(-3,4.8)$ & 0.969 \\
\hline & Hospital stay duration (day) & $5.0 \pm 0.6$ & $5.0 \pm 0.7$ & $-0.0(-0.2,0.1)$ & 0.924 \\
\hline \multirow[t]{3}{*}{ Fetal } & Fetal weight (g) & $2,974.8 \pm 594.8$ & $2,977.4 \pm 620.3$ & $-2.7(-144.0,138.6)$ & 0.970 \\
\hline & Apgar score $(1 \mathrm{~min})<7(\%)$ & $31(22.0)$ & $23(15.8)$ & $6.9(-2.1,15.9)$ & 0.178 \\
\hline & Apgar score $(5 \mathrm{~min})<7(\%)$ & $6(4.3)$ & $0(0)$ & $4.3(0.8,9)$ & $0.012 *$ \\
\hline
\end{tabular}

Values are presented as mean \pm SD or number (\%). SBP: systolic blood pressure, HR: heart rate, preoperative: before surgery, postoperative: 1 day after surgery, hct: hematocrit, dhct: mean difference of hct (preoperative hct-postoperative hct), EBL: Estimated blood loss. *P value < 0.05 , ${ }^{\dagger} \mathrm{P}$ value $<0.01$. 
[0\%], respectively, $\mathrm{P}=0.012$ ) (Table 2).

Postoperative hct levels were lower than the preoperative hematocrit levels in both groups, and the hct levels were lower on postoperative day (POD) 3 than on POD 1. The hct levels on POD 1 and POD 3 were significantly lower in the general anesthesia group than the spinal anesthesia group (Fig. 2).

\section{DISCUSSION}

Our results show that general anesthesia tends to cause more bleeding than spinal anesthesia, as the postoperative mean EBL volume and the mean difference between the pre- and postoperative hct level was larger with general anesthesia than with spinal anesthesia.

Although cesarean section is used to promote maternal health and fetal well-being, the maternal morbidity and mortality rates associated with this procedure remain high. The maternal morbidity rate associated with a cesarean section is approximately $35.7 \%$ [8]. Perioperative bleeding is the main cause of death related to cesarean section; the EBL volume that requires transfusion is about $1,000 \mathrm{ml}$ [9]. Maternal bleeding related to cesarean section is more common with general than regional anesthesia $[3,4]$. Increased maternal postoperative bleeding under general anesthesia than with regional anesthesia may be due to the uterine-relaxing effects of inhalation anesthetics [10].

Saygi et al. [11] performed a prospective randomized study comparing maternal and fetal outcomes between general and spinal anesthesia groups undergoing cesarean section. The postoperative hct levels $(29.9 \pm 3.2 \%$ vs. 32.2 $\pm 4.1 \%, \mathrm{P}=0.004$ ) were significantly lower in the general anesthesia group than in the spinal anesthesia group, similar to our results.

In this study, EBL was higher, and postoperative hematocrit levels were lower in the general anesthesia group than in the spinal anesthesia group. Moreover, the postoperative heart rate seemed to increase to compensate for hypovolemia or anemia in the general anesthesia group. Interestingly, the operation time was significantly longer in the general anesthesia group than the spinal anesthesia group, apparently due to an increased rate of operative manipulations to stop bleeding.

Guay [12] reported that regional anesthesia had a clear effect on surgical blood loss, but this did not usually reduce the number of transfused patients. Similarly, in this study, there was no significant difference in the number of transfused patients between the two groups.

In this study, postoperative hematocrit levels were significantly lower in the general anesthesia group than in the spinal anesthesia group, but they were significantly lower on POD 3 than on POD 1 (Fig. 2). Erythropoiesis was reportedly increased by day 7 after surgical blood loss, such that the postoperative hct deficit was corrected by day 28 [13].

The present study used the Apgar score as an indicator of fetal well-being. The Apgar score is a comprehensive measure of the clinical and cardiopulmonary functions of newborns. The proportion of newborns with 1-min Apgar scores $<7$ was not significantly different between the two groups, while the proportion with 5-min Apgar scores $<7$ was significantly larger in the general anesthesia group than the spinal anesthesia group (6/141 [4.3\%] vs. 0/146 [0\%], respectively, $\mathrm{P}=0.012$ ) (Table 2).

Recent studies $[3,14]$ reported no significant difference in

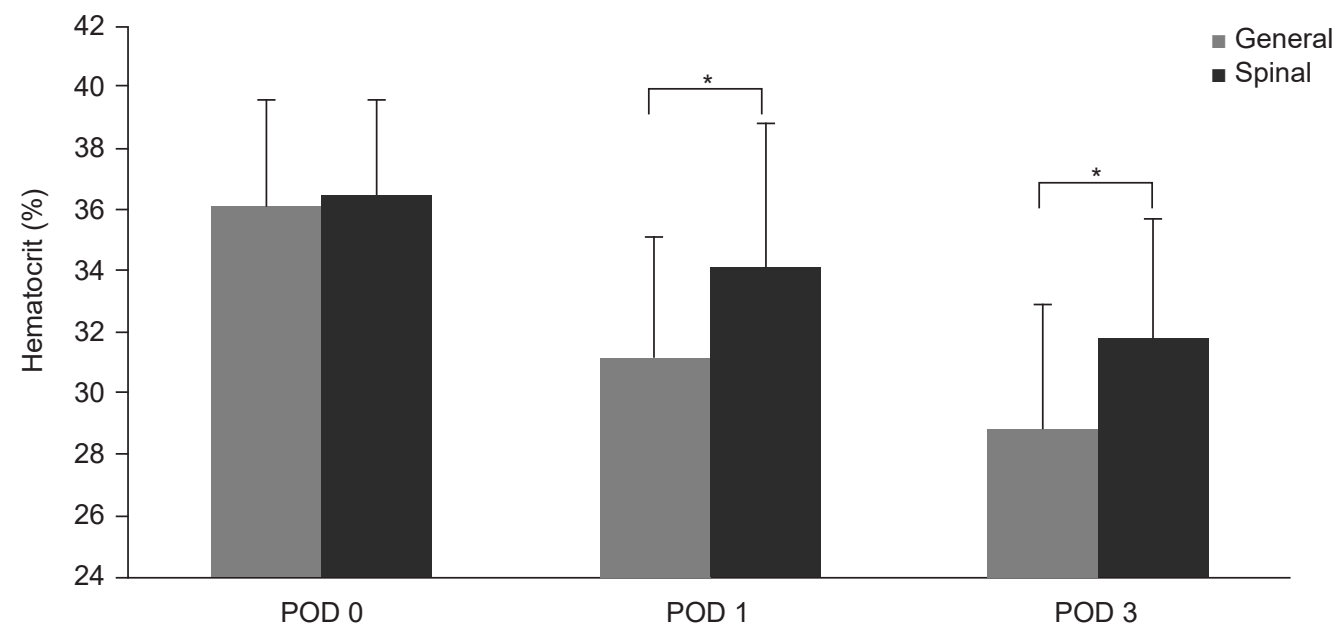

Fig. 2. Perioperative hematocrit (\%). POD 0: preoperative, POD 1: postoperative 1 day, POD 3: postoperative 3 days. *P $<0.01$. 
the 1- or 5-min Apgar scores of newborn babies under general versus spinal anesthesia for cesarean section. However, Tonni et al. [15] reported that, although the mother's oxygen partial pressure and saturation were higher with general anesthesia than with regional anesthesia, the partial pressure of oxygen and umbilical cord blood $\mathrm{pH}$ in the general anesthesia group were lower than in the spinal and epidural groups. They hypothesized that newborns delivered under general anesthesia experience transient respiratory depression because anesthetics given to the mother cross the placental barrier and enter fetal circulation.

In this study, the proportion with 5-min Apgar scores $<7$ was significantly larger in the general anesthesia group than the spinal anesthesia group. We supposed that anesthetic agents crossing the placenta might influence the fetus to some degree, although the fetus well tolerated them. Regional anesthesia can minimize the exposure of newborns to anesthetics and improve placental perfusion and oxygenation of the fetus due to sympathetic blockade. Therefore, regional anesthesia is preferable to general anesthesia during the cesarean section for both maternal and fetal safety.

Usually, the administration of general anesthesia is inevitable in cases of maternal coagulopathy or fetal distress. Neonatal respiratory depression accompanied by low Apgar scores and umbilical arterial and venous $\mathrm{pH}$ changes associated with general anesthesia is often transient. However, careful and appropriately administered general anesthetic has no significant adverse effects on fetuses or neonates [16].

Although many reports have shown that regional and general anesthesia are almost identical in terms of neonatal well-being, regional anesthesia, especially spinal, is recommended for elective cesarean section to avoid neonatal depression, especially for preterm delivery.

This study had some limitations. First, it was retrospective study, and we could not control all confounding variables that may have affected the outcomes. Second, the $\mathrm{P}$ values of some major results (Apgar scores) are relatively large; the sample size is relatively small. Therefore, the significant results might be purely by chance (random error). The sample size was based on the number of participants required to detect a statistically significant difference in the hct level, but not the Apgar score between the groups. Since spinal anesthesia for cesarean section was only introduced in our hospital two years ago, the maximum possible sample size for the spinal anesthesia group was 146 .
According to the power calculation, 232 subjects were needed in each group to detect statistically significant differences in Apgar scores. Thus, our findings regarding the Apgar scores should be interpreted with caution, and future research should include adequate sample sizes.

During cesarean section, general anesthesia group is associated with more maternal blood loss and a larger proportion of newborns with 5-min Apgar scores $<7$ than spinal anesthesia.

\section{CONFLICTS OF INTEREST}

No potential conflict of interest relevant to this article was reported.

\section{AUTHOR CONTRIBUTIONS}

Conceptualization: Young Seok Jee. Data curation: HwangJu You. Writing - original draft: Young Seok Jee. Statistical analysis: Tae-Yun Sung, Young Seok Jee. Writing - review \& editing: Tae-Yun Sung, Young Seok Jee, Choon-Kyu Cho.

\section{ORCID}

Tae-Yun Sung, https://orcid.org/0000-0002-0714-1477

Young Seok Jee, https://orcid.org/0000-0001-9154-0691

Hwang-Ju You, https://orcid.org/0000-0001-9921-4241

Choon-Kyu Cho, https://orcid.org/0000-0001-9906-1396

\section{REFERENCES}

1. Enkin M, Keirse MJ, Neilson J, Crowther C, Duley L, Hodnett E, et al. Effective care in pregnancy and childbirth: a synopsis. Birth 2001; 28: 41-51.

2. Ng K, Parsons J, Cyna AM, Middleton P. Spinal versus epidural anaesthesia for caesarean section. Cochrane Database Syst Rev 2004; (2): CD003765.

3. Dyer RA, Els I, Farbas J, Torr GJ, Schoeman LK, James MF. Prospective, randomized trial comparing general with spinal anesthesia for cesarean delivery in preeclamptic patients with a nonreassuring fetal heart trace. Anesthesiology 2003; 99: 561-9; discussion 5A-6A.

4. Hong JY, Jee YS, Yoon HJ, Kim SM. Comparison of general and epidural anesthesia in elective cesarean section for placenta previa totalis: maternal hemodynamics, blood loss and neonatal outcome. Int J Obstet Anesth 2003; 12: 12-6.

5. Afolabi BB, Lesi FE. Regional versus general anaesthesia for 
caesarean section. Cochrane Database Syst Rev 2012; (10): CD004350.

6. Mancuso A, De Vivo A, Giacobbe A, Priola V, Maggio Savasta L, et al. General versus spinal anaesthesia for elective caesarean sections: effects on neonatal short-term outcome. A prospective randomised study. J Matern Fetal Neonatal Med 2010; 23: 1114-8.

7. Sener EB, Guldogus F, Karakaya D, Baris S, Kocamanoglu S, Tur A. Comparison of neonatal effects of epidural and general anesthesia for cesarean section. Gynecol Obstet Invest 2003; 55: 41-5.

8. Ronsmans C, Graham WJ; Lancet Maternal Survival Series steering group. Maternal mortality: who, when, where, and why. Lancet 2006; 368: 1189-200.

9. Bergholt T, Stenderup JK, Vedsted-Jakobsen A, Helm P, Lenstrup C. Intraoperative surgical complication during cesarean section: an observational study of the incidence and risk factors. Acta Obstet Gynecol Scand 2003; 82: 251-6.

10. Wong CA. General anesthesia is unacceptable for elective cesarean section. Int J Obstet Anesth 2010; 19: 209-12.
11. Saygı Aİ, Özdamar Ö, Gün İ, Emirkadı H, Müngen E, Akpak YK. Comparison of maternal and fetal outcomes among patients undergoing cesarean section under general and spinal anesthesia: a randomized clinical trial. Sao Paulo Med J 2015; 133: 227-34.

12. Guay J. The effect of neuraxial blocks on surgical blood loss and blood transfusion requirements: a meta-analysis. J Clin Anesth 2006; 18: 124-8.

13. Wallis JP, Wells AW, Whitehead S, Brewster N. Recovery from post-operative anaemia. Transfus Med 2005; 15: 413-8.

14. Kavak ZN, Başgül A, Ceyhan N. Short-term outcome of newborn infants: spinal versus general anesthesia for elective cesarean section. A prospective randomized study. Eur J Obstet Gynecol Reprod Biol 2001; 100: 50-4.

15. Tonni G, Ferrari B, De Felice C, Ventura A. Fetal acid-base and neonatal status after general and neuraxial anesthesia for elective cesarean section. Int J Gynaecol Obstet 2007; 97: 143-6.

16. Mattingly JE, D'Alessio J, Ramanathan J. Effects of obstetric analgesics and anesthetics on the neonate: a review. Paediatr Drugs 2003; 5: 615-27. 